\section{Aplicación intravesical de toxina botulínica y su repercusión en la calidad de vida de pacientes con lesión medular y vejiga neurogénica. Experiencia institucional}

Durán-Ortiz S, ${ }^{1}$ García-Herrera DA, ${ }^{1}$ Pérez-Hernández BO, ${ }^{2}$ Pérez-Zavala R, ${ }^{2}$ León $\mathrm{SR}^{2}$

Resumen

ANTECEDENTES: la vejiga neurogénica es un padecimiento común en pacientes con lesión medular, que provoca efectos devastadores y gran repercusión en la calidad de vida.

OBJETIVO: determinar la calidad de vida en pacientes con lesión medular y vejiga neurogénica después de la aplicación intravesical de toxina botulínica.

MATERIALES Y MÉTODOS: estudio descriptivo y prospectivo efectuado en pacientes con lesión medular y vejiga neurogénica de alta presión y disinergia vesicoesfinteriana comprobada mediante estudio urodinámico. Se realizó una encuesta antes de la aplicación, un mes y tres meses después de la administración de la toxina botulínica. Se evaluaron aspectos sociales, laborales, psicológicos y funcionales. Se efectuó el estudio urodinámico luego de 3 meses de la aplicación de la toxina.

RESULTADOS: se registraron 16 pacientes con lesión medular y vejiga neurogénica; los cambios en los parámetros del cuestionario que evaluaron la calidad de vida al mes y tres meses posaplicación, en comparación con la valoración preaplicación fueron estadísticamente significativos. En todos los pacientes se observó un aumento en la capacidad vesical (20-80 mL). Esta variable no se relacionó con la edad, sexo, ni tiempo de evolución de la lesión. Se observó que a menor capacidad vesical antes de la aplicación de la toxina, mayor aumento en la capacidad vesical en el estudio urodinámico de control.

CONCLUSIONES: la aplicación intravesical de toxina botulínica mejora la calidad de vida de pacientes con lesión medular y vejiga neurogénica; además, disminuye las complicaciones urológicas crónicas y representa un procedimiento de baja morbilidad y mortalidad.

PALABRAS CLAVE: lesión medular, vejiga neurogénica, disinergia vesicoesfinteriana, toxina botulínica.

\footnotetext{
${ }^{1}$ Departamento de Urología.

${ }^{2}$ Departamento de Rehabilitación Neurológica. Instituto Nacional de Rehabilitación, Ciudad de México.
}

Recibido: enero 2017

Aceptado: octubre 2017

Correspondencia

David Alejandro García Herrera

d.alejandro.garcia.herrera@gmail.com

Este artículo debe citarse como

Durán-Ortiz S, García-Herrera DA, Pérez-Hernández BO, Pérez-Zavala R, León SR. Aplicación intravesical de toxina botulínica y su repercusión en la calidad de vida de pacientes con lesión medular y vejiga neurogénica. Experiencia institucional. Rev Mex Urol. 2017 nov-dic;77(6):453-463.

DOI: https://doi.org/10.24245/revmexurol.v77i6.1179 


\section{Impact of intravesical application of botulinum toxin on quality of life of patients with spinal cord injury and neurogenic bladder: Experience at a national rehabilitation center}

Durán-Ortiz S, ${ }^{1}$ García-Herrera DA, ${ }^{1}$ Pérez-Hernández BO, ${ }^{2}$ Pérez-Zavala $\mathrm{R},{ }^{2}$ León $\mathrm{SR}^{2}$

\begin{abstract}
BACKGROUND: Neurogenic bladder is a common disorder in patients with spinal cord injury. Its effects are devastating, profoundly impacting the quality of life of those patients.
\end{abstract}

AIM: To determine the effect of botulinum toxin application on the quality of life of patients with spinal cord injury and neurogenic bladder.

MATERIALS AND METHODS: A descriptive, prospective analysis was conducted on patients with spinal cord injury, high pressure neurogenic bladder, and vesicosphincter dyssynergia corroborated through urodynamic study. A questionnaire was applied before botulinum toxin application, and again, one and three months after application, covering social, occupational, psychologic, and functional aspects. Urodynamic study was performed at a minimum of 3 months after botulinum toxin application.

RESULTS: Sixteen patients with spinal cord injury and neurogenic bladder were enrolled in the study. There were statistically significant changes in the questionnaire parameters evaluating quality of life at one and three months after the procedure, compared with the preapplication results. All patients had an increase in bladder capacity (20-80 mL), regardless of patient age, sex, or time with spinal cord injury. Patients with a lower bladder capacity before botulinum toxin application had a higher increase in bladder capacity in the control urodynamic study.

CONCLUSIONS: Intravesical botulinum toxin application improves the quality of life in patients with spinal cord injury and neurogenic bladder, reducing chronic urologic complications, in addition to being a procedure with low morbidity and mortality.

KEYWORDS: Spinal cord injury; Neurogenic bladder; Vesicosphincter dyssynergia; Botulinum toxin

\footnotetext{
${ }^{1}$ Departamento de Urología.

2 Departamento de Rehabilitación Neurológica.

Instituto Nacional de Rehabilitación, Ciudad de México.

Correspondence

David Alejandro García Herrera

d.alejandro.garcia.herrera@gmail.com
} 


\section{ANTECEDENTES}

La vejiga neurogénica es un término aplicado a la disfunción de la vejiga y del esfínter urinario, provocada por alguna alteración neurológica o traumatismo. Los trastornos asociados con la vejiga neurogénica incluyen enfermedades degenerativas (esclerosis múltiple, enfermedad de Parkinson), alteraciones con afección neurológica a largo plazo (diabetes mellitus, anemia perniciosa, tabes dorsal) o padecimientos asociados con severa disfunción neurológica (enfermedad cerebrovascular, espina bífida, mielomeningocele, tumores del sistema nervioso central, traumatismo y lesión medular). ${ }^{1}$ La incidencia de lesión medular en Estados Unidos es de 10,000 casos nuevos por año, de los que $51 \%$ ocurre en individuos de entre 16 y 30 años de edad (promedio de 32.6 años), con mayor afectación en hombres (76.8\%) que en mujeres (23.2\%). En México se ha registrado una incidencia de 18.1 por cada millón de habitantes. ${ }^{2,3}$

El comportamiento de la vejiga neurogénica en pacientes con lesión medular depende del nivel y complejidad de la lesión. Las manifestaciones varían desde síntomas del conducto urinario bajo (urgencia, frecuencia e incontinencia urinaria, incluso dificultad para completar la micción) hasta retención urinaria completa e imposibilidad para el vaciamiento vesical. ${ }^{2}$ Puede deberse a una lesión de la neurona motora superior (por arriba de S2) o inferior. Las estructuras del conducto urinario inferior reciben triple inervación estimulada por el sistema nervioso simpático, parasimpático y somático. El núcleo simpático se localiza en la médula espinal dorso-lumbar, desde las metámeras T10 a L2. A través del nervio hipogástrico inhibe al músculo detrusor y al mismo tiempo estimula la contracción del esfínter uretral interno. El núcleo parasimpático se sitúa del segundo al cuarto espacio sacro de la médula espinal y de éste parte el nervio pélvico; este sistema estimula la contracción del músculo detrusor de la vejiga. El núcleo somático, o de Onuf, se ubica en el espacio sacro S3-S4, a través del nervio pudendo, donde inerva al esfínter uretral externo, que se constituye como un elemento importante de la continencia urinaria. La vía urinaria inferior se encuentra en control voluntario por los centros superiores cerebrales, donde el área motora del detrusor y el núcleo pontino son los responsables del control consciente y voluntario de la micción, conexión que se interrumpe en pacientes con lesiones medulares. ${ }^{2,4}$

La Sociedad Internacional de Continencia definió, en 2002, la vejiga hiperactiva como la urgencia miccional asociada con aumento de la frecuencia urinaria y nicturia relacionada o no con incontinencia de urgencia. La vejiga hiperactiva puede tener un origen neurogénico (hiperreflexia del detrusor) o no neurogénico (inestabilidad vesical), este último es el más frecuente. ${ }^{5,6}$

El estudio urodinámico confirma la hiperreflexia del detrusor y la coexistencia o no de disinergia vesicoesfinteriana.

La vejiga hiperactiva de origen neurogénico es frecuente en pacientes con lesión medular. La incontinencia urinaria de urgencia relacionada con vejiga hiperactiva severa influye de manera negativa en la calidad de vida del paciente, evita su reintegración al ámbito social. ${ }^{6}$

El tratamiento de primera línea consiste en fármacos anticolinérgicos; sin embargo, debido a los múltiples efectos adversos y su costo elevado disminuye el apego al tratamiento. En la actualidad la aplicación intravesical de toxina botulínica ha demostrado eficacia en pacientes con vejiga hiperactiva de origen neurogénico y no neurogénico. ${ }^{7-9}$

La toxina botulínica contiene una fracción neurotóxica que consiste en una proteína con peso molecular de 150,000 daltons, que produce parálisis a través de una unión irreversible con la 
terminal nerviosa colinérgica presináptica, que resulta en interrupción del flujo de calcio iónico. ${ }^{10} \mathrm{El}$ efecto final es la quimiodenervación temporal en la unión neuromuscular que no produce ninguna lesión física en las estructuras nerviosas; sin embargo, la parálisis puede provocar asfixia o graves lesiones neurológicas por anoxia, al bloquear la función respiratoria con dosis excesivas. Actualmente se reconocen 8 tipos de toxina botulínica, entre éstas, la A, B y E se han relacionado con el botulismo en humanos. ${ }^{11}$

En la práctica urológica se prescriben preparaciones comerciales de toxina botulínica tipo A para el tratamiento de lesiones medulares con disinergia vésicoesfinteriana, con la finalidad de aumentar la capacidad vesical y disminuir la contractilidad del detrusor, para de esta manera iniciar un programa de cateterismo vesical intermitente..$^{6,10,11}$ El cateterismo intermitente permite al paciente el vaciamiento urinario completo, programado y limpio, con la finalidad de evitar episodios de incontinencia urinaria y disminuir el riesgo de complicaciones, además de la reintegración a la vida social y sexual. Los resultados son satisfactorios en la mayoría de los casos, con mínimas complicaciones sistémicas o locales. La eficacia máxima de la aplicación de toxina botulínica tipo A se produce entre siete y treinta días posaplicación, con efecto de hasta 6 meses. ${ }^{12-14}$ La técnica de aplicación de la toxina botulínica varía entre los diferentes grupos de trabajo y se considera un procedimiento ambulatorio. La frecuencia recomendada entre una infiltración y otra es de 3 a 4 meses. ${ }^{13,15,16}$

El objetivo de este estudio fue: determinar la calidad de vida en pacientes con lesión medular y vejiga neurogénica después de la aplicación intravesical de toxina botulínica.

\section{MATERIALES Y MÉTODOS}

Estudio descriptivo, prospectivo y experimental, en el que participaron los servicios de Urolo- gía y Rehabilitación Neurológica del Instituto Nacional de Rehabilitación de la Ciudad de México. Criterios de inclusión: pacientes de ambos sexos, de entre 15 y 75 años de edad, con diagnóstico de lesión medular en cualquier nivel neurológico y origen, con más de 6 meses de evolución, vejiga neurogénica hiperrefléxica y disinergia vesicoesfinteriana confirmadas mediante estudio urodinámico. Criterios de exclusión: pacientes con complicaciones médica-urológicas diferentes a las anteriores (litiasis vesical, bacteriuria sintomática, estenosis uretral), que impidieran o contraindicaran la aplicación intravesical de toxina botulínica; pacientes en tratamiento que interfiriera la aplicación de la toxina, quienes tuvieran algún impedimento físico o mental que les imposibilitara efectuar cateterismos intermitentes u otra alteración neurológica adquirida que provocara vejiga neurogénica.

\section{Técnica de aplicación intravesical de toxina botulínica}

En el quirófano, con administración de anestesia regional, se realiza la aplicación intravesical de la toxina botulínica con técnica estéril, mediante cistoscopio de $21 \mathrm{Fr}$ y aguja de Williams con técnica en espiral; con aplicación radiada en el esfínter uretral externo, colocación de sonda Foley $16 / 18 \mathrm{Fr}$ transuretral y estancia hospitalaria para observación durante 24 horas. Luego de 7 días del egreso hospitalario se programa una cita médica de revisión. Antes de la aplicación, durante el procedimiento y posterior al mismo, los pacientes reciben tratamiento con antibióticos con quinolonas (levofloxacina).

Dilución de la toxina botulínica. Se utiliza toxina botulínica tipo A (Botox ${ }^{\circledR}$ ). Cada frasco contiene 100 unidades de toxina botulínica, que se diluyen en $8 \mathrm{~mL}$ de solución fisiológica; posteriormente se mezcla (delicadamente), se toma la dilución con una jeringa de 20 cc y se 

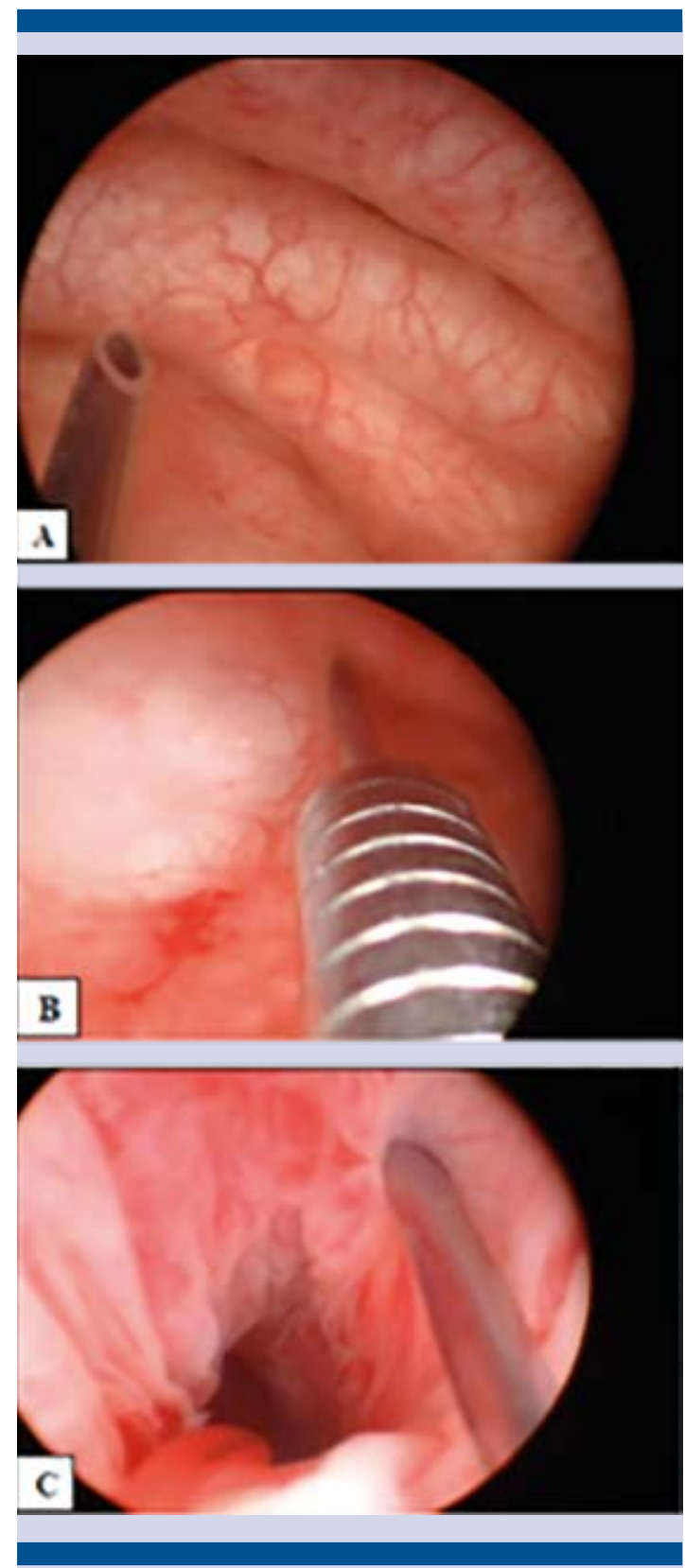

Figura 1. Cistoscopia: aguja para aplicación de la toxina botulínica (A), aplicación en el domo vesical (B) y en el esfínter (C).

aplican 15 cc. Para esta técnica se utilizaron tres frascos de toxina botulínica (300 U).
Instrumento de evaluación de la calidad de vida. Se utilizó el Cuestionario de Repercusión de la Incontinencia (CII) en su versión corta (adaptado del Incontinence Impact Questionnaire IIQ-7) del Grupo de Investigación del Programa de Continencia para Mujeres (Figura 2), que contiene 7 preguntas que evalúan la repercusión en la calidad de vida del paciente, tomando en cuenta aspectos como: actividad física, viajes y traslados, relaciones personales-sociales y aspecto emocional. Los parámetros para calificar la calidad de vida se basan en 4 posibles respuestas: en absoluto (1), un poco (2), moderadamente (3) y mucho (4), con lo que se obtiene un puntaje para clasificar la repercusión-efecto de la incontinencia en la calidad de vida en leve (1-7 puntos), moderada (8-15 puntos) o severa (16 o más puntos). El cuestionario se aplicó antes de la administración de la toxina botulínica y, posteriormente, a los 3 y 6 meses de la misma, además de efectuar el estudio urodinámico de control en cada paciente.

\section{Análisis estadístico}

Los datos se analizaron con el programa SPSS versión 15. Se utilizó estadística descriptiva, mediante medidas de tendencia central, proporción y dispersión; también se implementó la prueba de $\mathrm{t}$ de Student para muestras relacionadas y $\mathrm{t}$ de Wilcoxon según su distribución. Se consideró estadísticamente significativo el valor de $p<$ 0.05 , además de tomar en cuenta un tamaño de muestra de 16 pacientes $(n=16)$ para tener un poder estadístico de $80 \%$.

\section{RESULTADOS}

Se evaluaron 16 pacientes con diagnóstico de lesión medular. Un paciente fue eliminado del estudio por problemas médicos que no le permitieron acudir a la última cita para completar el estudio urodinámico de control y responder el último cuestionario. 


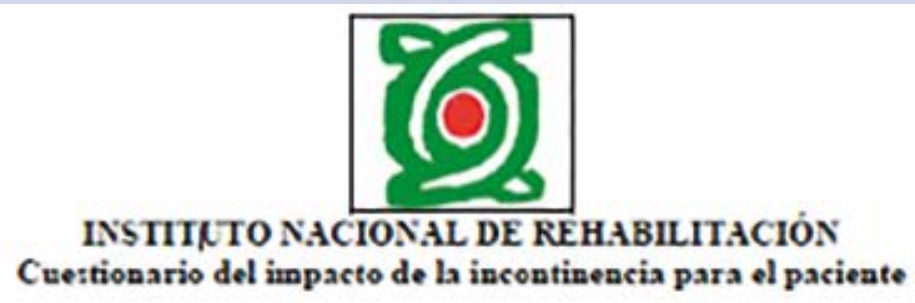

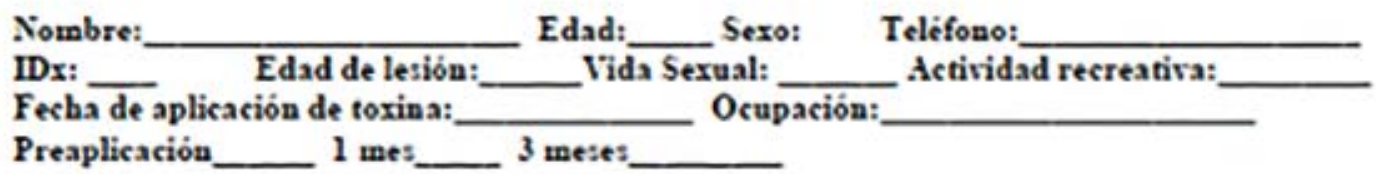

Algunas personas creen que la pérdida involuntaria de orina puede afectar sus actividades, relacione: $y$ sentimientos. Las preguntas que aparecen a continuación se refierea a jreas de su vida que puedea verse influenciadas o alteradas por este problema. Para cada pregunta, encierre en un circulo la respuesta que describe mejor cuintas de sus actividades, relaciones y rentimientos se ven afestadas por la pérdida involuntana de orina.

Cuinto ha afectado la pézdida involuntaria de orina su...

1. ¿Capacidad para realizar los quebaceres domésticos (cocinar, limpiar, lavar)?
En absoluto
Un poco
Moderadamente
Mucho

2. Actividad fisica como hacer terapias u otros ejercicios?
En absoluto
Un poco
Moderadamente
Mucho

3. Actividades de entretenimiento (cine, conciertos, etc.)?

En absoluto Un poco Moderadamente Mucho

4. ¿Capacidad par tiajar en automóvil o autobus más de 30 minutos de:de su ca:a?

En absoluto Un poco Moderadamente Mucho

5. ¿Paticipación en actividades sociales fuera de casa?

En absoluto Un poco Moderadamente Mucho

6. ¿Salud emocionsl (nerviosismo, depresión, etc.)?

En absoluto Un poco Moderadamente Mucho

7. La pérdida involuntania de orina ¿le ba becho sentirse frustrado?

En absoluto Un poco Moderadamente Mucho

Figura 2. Cuestionario de Repercusión de la Incontinencia (adaptado de la versión corta IIQ-7 [Incontinence Impact Questionnaire-Short Form IIQ-7]).

La edad promedio fue de 31.5 años ( $\mathrm{DE} \pm 13.8$;

límites mínimo y máximo de 19 a 79 años, res- pectivamente) y el tiempo de evolución de la lesión de 38.6 meses ( $\mathrm{DE} \pm 26.9$; 11-96 meses). 
Se registraron 11 (68.8\%) hombres y 5 (31.2\%) mujeres.

La localización de las lesiones se distribuyó de la siguiente manera: cervical $3(18.8 \%)$ casos, torácico 12 (75\%) y lumbar 1 (6.2\%); 11 (68.8\%) de los pacientes correspondieron a lesión medular completa (escala A de acuerdo con el ASIA) y el resto a lesión incompleta (Cuadro 1).

La capacidad vesical después de la aplicación de la toxina botulínica tuvo un incremento significativo $(p=0.0001)$ : antes de la aplicación (medida por urodinamia) fue de $136.6 \mathrm{~mL}(\mathrm{DE} \pm 21.7 \mathrm{~mL})$ y luego de 3 meses de $237.4 \mathrm{~mL}(\mathrm{DE} \pm 87.3 \mathrm{~mL}$ ).

Respecto del cambio en la capacidad vesical por el tratamiento y modificaciones en la escala del CII, se encontró que antes de la aplicación la correlación entre el puntaje global del CIl y la capacidad vesical era rho $=0.03, p=0.77$ (ninguna correlación hasta ese momento), luego de 1 mes de tratamiento: rho $=-0.644, p=$ 0.007 (correlación media-alta) y después de tres meses la correlación ascendió a rho $=-0.648$, $\mathrm{p}=0.009$. Los resultados indican que cuanto mayor es el cambio logrado en la capacidad vesical postratamiento respecto de la capacidad pretratamiento, se obtienen mejores respuestas

Cuadro 1. Características de los pacientes con lesión medular y vejiga neurogénica

\begin{tabular}{|l|c|c|c|}
\hline Variable & & n & $\%$ \\
\hline \multirow{2}{*}{ Sexo } & Masculino & 11 & 68.8 \\
\hline \multirow{2}{*}{ Estado civil } & Femenino & 5 & 31.2 \\
\hline \multirow{3}{*}{ Localización de la lesión } & Soltero & 12 & 75 \\
\hline & Casado & 4 & 25 \\
\hline \multirow{2}{*}{ Tipo de lesión } & Cervical & 3 & 18.8 \\
\hline & Lumbar & 12 & 75 \\
\hline & Completa & 11 & 6.2 \\
\hline & Incompleta & 5 & 31.2 \\
\hline
\end{tabular}

en el nivel 1 de la escala del Cll después de 1 y 3 meses de la aplicación de la toxina botulínica (Figuras 3, 4 y 5). Los cambios en la escala del Cll se dieron a expensas de la mejoría en las dimensiones de salud emocional y frustración.

La comparación entre sexo $(p=0.56)$, edad ( $p$ $=0.55)$ y tiempo de evolución de la lesión ( $p$ $=0.17)$ no mostró diferencia estadísticamente

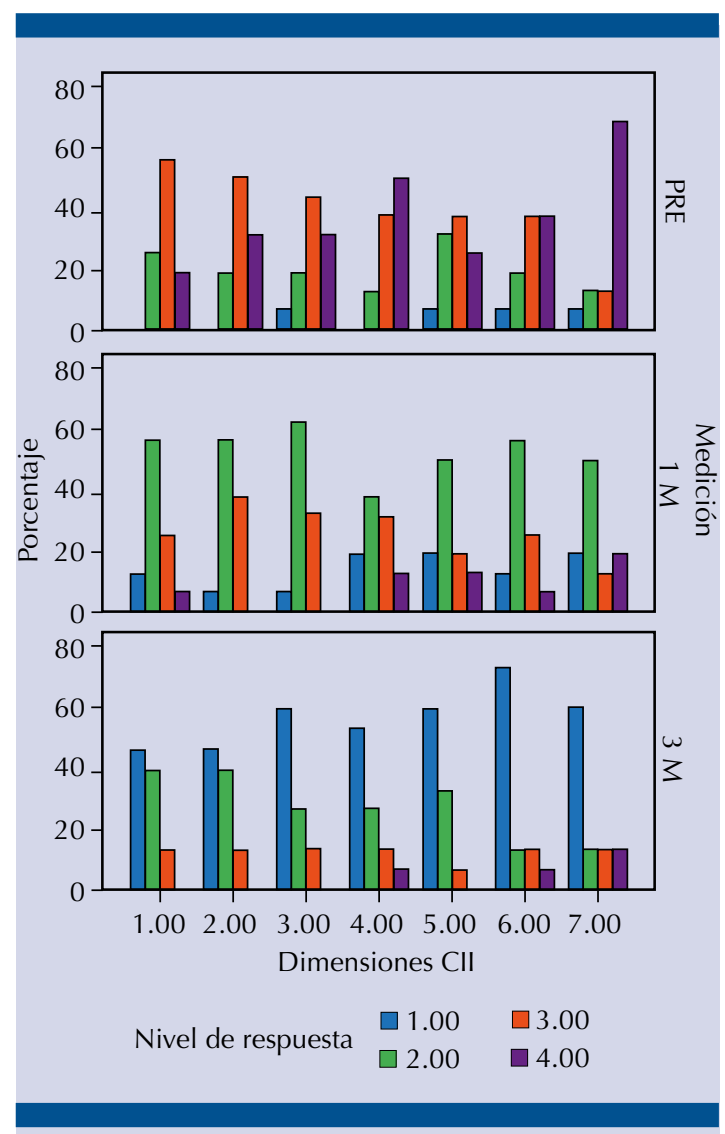

Figura 3. Cambios en los niveles de respuesta del Cuestionario de Repercusión de la Incontinencia (CII) desde la medición preaplicación, luego de un mes y a los tres meses (los cambios se encontraron con la prueba de Friedman para $\mathrm{K}$ muestras relacionadas en cada dimensión del cuestionario). Se destaca la prevalencia en los niveles 4 [guinda] de respuesta pretratamiento versus los niveles 2 [verde] al mes de tratamiento y los niveles 1 [azul] a los tres meses de tratamiento. 


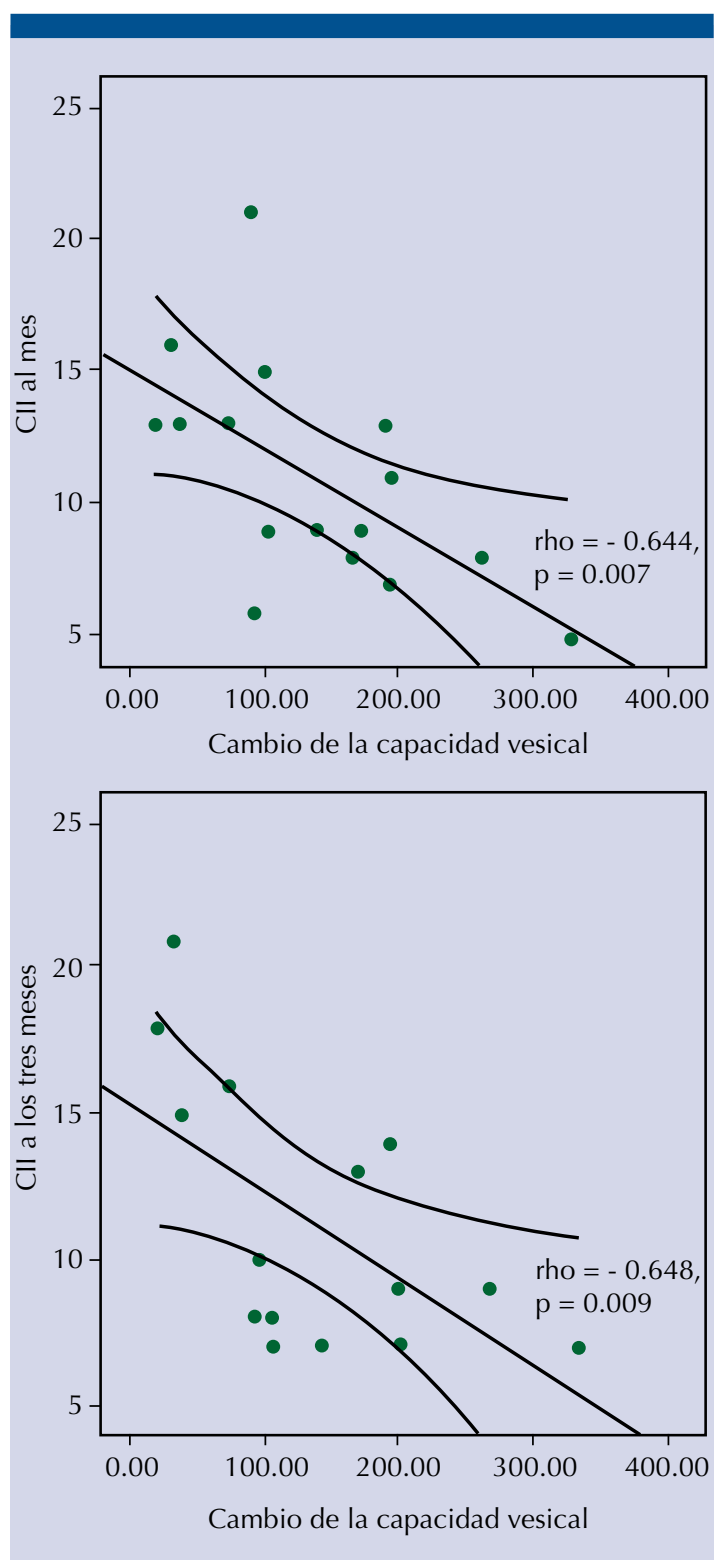

Figura 4. Correlación entre el cambio en la capacidad vesical por el efecto del tratamiento y los cambios en la escala del Cuestionario de Repercusión de la Incontinencia (CII).

significativa en la capacidad vesical después de la aplicación de la toxina botulínica (Cuadro 2).

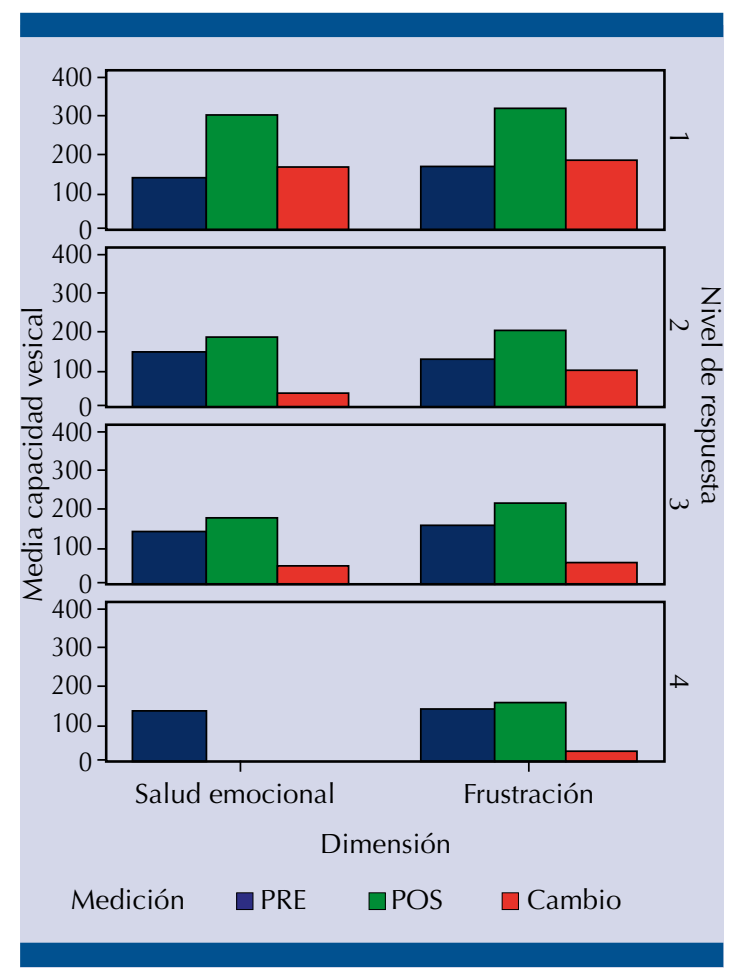

Figura 5. Relación entre el cambio en la capacidad vesical y los parámetros del Cuestionario de Repercusión de la Continencia (CII).

En relación con la edad, se encontró que el cambio en la capacidad vesical fue más notable en los pacientes mayores de 32 años, pues iniciaron con capacidad vesical muy baja (119.5 $\mathrm{mL})$ y terminaron con capacidad alta $(290 \mathrm{~mL})$; en contraste, los jóvenes comenzaron con menor deterioro de la capacidad vesical y por ello el cambio no fue tan evidente (Cuadro 3).

Respecto del tiempo de evolución de la lesión, se observó que los pacientes con 11 a 24 meses de evolución tuvieron mayor cambio en la capacidad vesical que quienes tuvieron 25 a 54 meses (Cuadro 4).

Después de analizar las variables relacionadas con la calidad de vida se encontró que $50 \%$ de los pacientes reportó no tener vida sexual activa; 
Cuadro 2. Relación entre el cambio en la capacidad vesical y sexo del paciente

\begin{tabular}{|c|c|c|}
\multirow{2}{*}{ Sexo (cambio mL) } & \multicolumn{2}{|c|}{ Capacidad vesical } \\
\cline { 2 - 3 } & Preaplicación & Posaplicación \\
\hline \multirow{2}{*}{ Masculino 127.3 } & $141.7 \mathrm{~mL}$ & $269 \mathrm{~mL}$ \\
& $(\mathrm{DE} \pm 21.3)$ & $(\mathrm{DE} \pm 78.1)$ \\
\hline \multirow{2}{*}{ Femenino 157.7} & $125.4 \mathrm{~mL}$ & $283.1 \mathrm{~mL}$ \\
$\mathrm{p}$ & $(\mathrm{DE} \pm 20.5)$ & $(\mathrm{DE} \pm 114.7)$ \\
\hline
\end{tabular}

Cuadro 3. Relación entre el cambio en la capacidad vesical y edad del paciente

\begin{tabular}{|c|c|c|}
\hline \multirow{2}{*}{$\begin{array}{l}\text { Edad (años): } \\
\text { cambio (mL) }\end{array}$} & \multicolumn{2}{|c|}{ Capacidad vesical } \\
\hline & Preaplicación & Posaplicación \\
\hline 19-25: 98.5 & $\begin{array}{c}135.2 \mathrm{~mL} \\
(\mathrm{DE} \pm 11.5)\end{array}$ & $\begin{array}{c}233.7 \mathrm{~mL} \\
(\mathrm{DE} \pm 66.2)\end{array}$ \\
\hline 26-32: 139.2 & $\begin{array}{c}145.8 \mathrm{~mL} \\
(\mathrm{DE} \pm 25.6)\end{array}$ & $\begin{array}{c}285 \mathrm{~mL} \\
(\mathrm{DE} \pm 85.0)\end{array}$ \\
\hline$>32: 170.5$ & $\begin{array}{c}119.5 \mathrm{~mL} \\
(\mathrm{DE} \pm 8.3)\end{array}$ & $\begin{array}{c}290 \mathrm{~mL} \\
(\mathrm{DE} \pm 118.6)\end{array}$ \\
\hline $\mathrm{p}$ & 0.13 & 0.60 \\
\hline
\end{tabular}

Cuadro 4. Relación entre el cambio en la capacidad vesical y tiempo con la lesión

\begin{tabular}{|l|c|c|}
\hline \multirow{2}{*}{$\begin{array}{l}\text { Tiempo con la lesión } \\
\text { (meses): cambio }(\mathrm{mL})\end{array}$} & \multicolumn{2}{|c|}{ Capacidad vesical } \\
\cline { 2 - 3 } & Preaplicación & Posaplicación \\
\hline 11-24 meses: 164.7 & $144.5 \mathrm{~mL}$ & $309.2 \mathrm{~mL}$ \\
& $(\mathrm{DE} \pm 21.2)$ & $(\mathrm{DE} \pm 88.3)$ \\
\hline $25-54$ meses: 77.4 & $130.6 \mathrm{~mL}$ & $208 \mathrm{~mL}$ \\
& $(\mathrm{DE} \pm 24.19$ & $(\mathrm{DE} \pm 76.3)$ \\
\hline Más de 54 meses: 162.3 & $130.2 \mathrm{~mL}$ & $292.5 \mathrm{~mL}$ \\
\hline$p$ & $(\mathrm{DE} \pm 20.5)$ & $(\mathrm{DE} \pm 64.4)$ \\
\hline$p$ & 0.46 & 0.77 \\
\hline
\end{tabular}

$9(56.3 \%)$ refirieron realizar alguna actividad recreativa y $5(31.2 \%)$ tenían empleo.

En cuanto a la percepción del paciente de su calidad de vida antes de la aplicación de la toxina se encontró que estaba muy limitada para involucrarse en distintas actividades. La mayoría de los pacientes expresó un grado importante de frustración $(68.7 \%) ; 6(37.5 \%)$ reportaron que la incontinencia condicionaba en forma negativa su salud emocional y otros $6(37.5 \%)$ de manera moderada. En la segunda encuesta (al mes posaplicación) se observó un cambio positivo en la calidad de vida, pues 9 (56.2\%) calificaron la mayor parte de los rubros "un poco" o "en absoluto"; mientras que $18.7 \%$ llegó a calificar una limitación importante para desarrollar sus actividades cotidianas.

Los cambios expresados en las dimensiones (ítems) del cuestionario que evaluaron la calidad de vida luego de 1 y 3 meses postratamiento fueron estadísticamente significativos $(p<0.01)$

\section{(Cuadro 5).}

Finalmente, al estudiar la correlación entre el cambio en la capacidad vesical y los resultados de la escala del CII, la valoración preaplicación no mostró correlación ( $p=0.77$ ); sin embargo, después de 1 mes de la aplicación de la toxina se encontró correlación alta-media $(p=0.007)$ y a los 3 meses una correlación alta $(p=0.009)$.

Ningún paciente tuvo complicaciones después de la aplicación de la toxina botulínica.

\section{DISCUSIÓN}

Todos los pacientes con lesión medular, incluso los que preservan sus funciones sensitivas $y$ motoras, manifiestan alteraciones en la función vesical, con afectación en la vía urinaria superior e incontinencia urinaria, lo que limita de forma importante su calidad de vida. Existen pocos estudios relacionados con el beneficio de la aplicación intravesical de la toxina botulínica; por tanto, esta investigación se efectuó como precedente de su aplicación y repercusión en la calidad de vida, dándole significación.

En la encuesta previa a la aplicación de la toxina botulínica, la mayoría de los pacientes reportó 
Cuadro 5. Cambios (promedio) durante la aplicación del Cuestionario de Repercusión de la Incontinencia (CII)

\begin{tabular}{|l|c|c|c|c|c|}
\hline Variable & $\mathbf{n}$ & Mínimo & Máximo & Media & Desv. típ. \\
\hline CII Pretratamiento & 16 & 14 & 28 & 21.75 & 4.68330 \\
CII al mes & 16 & 10 & 26 & 16 & 4.19524 \\
Cll a los 3 meses & 15 & 7 & 21 & 11.26 & 4.57426 \\
p de Friedman & & & & 0.0001 & \\
\hline
\end{tabular}

un grado importante de frustración y repercusión negativa al realizar sus actividades cotidianas, su vida social y salud emocional. Durante el seguimiento se observaron cambios positivos en la calidad de vida e incremento en la capacidad vesical. Luego de tres aplicaciones todos se encontraban en un programa de cateterismo limpio intermitente, ya sea de forma independiente o con ayuda, en su domicilio. No se registraron complicaciones, infecciones, episodios de incontinencia o efectos adversos relacionados con la aplicación de la toxina botulínica.

En la tercera entrevista se realizó la última encuesta del Cll y se decidió tener una medida objetiva (cuantitativa) de la evolución de los pacientes mediante el estudio urodinámico de control. Todos los pacientes mostraron un cambio positivo en mayor o menor grado al completar el cuestionario, que se reflejó en mejor calidad de vida al disminuir la incontinencia, debido al incremento en la capacidad vesical. Este dato coincide con lo reportado en la bibliografía acerca de los efectos benéficos de la toxina botulínica intravesical, cuyo tiempo de acción promedio es de dos semanas. De esta manera, al disminuir o curar la continencia urinaria, los pacientes pueden desempeñarse en actividades cotidianas y mejorar su calidad de vida.

Uno de los objetivos de este estudio fue buscar criterios que permitieran estandarizar el uso de la toxina botulínica intravesical en los pacientes con lesión medular; sin embargo, no obtuvimos los resultados deseados para llegar a esta conclusión, pues el tiempo de evolución de la lesión no tuvo relación significativa con el cambio en la capacidad vesical; por tanto, debe tomarse con reserva la hipótesis de que a mayor tiempo de lesión medular menor será el efecto del tratamiento. En cuanto a la edad del paciente, se observó que a mayor edad (> 32 años) mejor efecto de la toxina; de esta manera, se asume que el tratamiento es útil en los pacientes de mayor edad y con menor capacidad vesical previa. Independientemente de los mililitros ganados en la capacidad vesical posaplicación de la toxina, los pacientes manifestaron mejor calidad de vida, que puede calificarse como subjetiva, pero es totalmente comprobable y medible por el estudio urodinámico de control. Se registró mejoría en la calidad de vida, como reflejo del inicio del programa de cateterismo intermitente, además del incremento en la capacidad vesical y disminución de los episodios de incontinencia.

Los hallazgos encontrados en este estudio resultan alentadores para continuar con la aplicación intravesical de toxina botulínica en pacientes con vejiga hiperactiva provocada por lesión medular, atendidos en el Instituto Nacional de Rehabilitación.

Las limitaciones del estudio fueron: tamaño reducido de la muestra, periodo de seguimiento corto y pérdida de uno de los pacientes. Y a pesar del número alto de pacientes con diagnóstico de lesión medular, no todos tenían vejiga de alta presión con disinergia vesicoesfinteriana. 


\section{CONCLUSIONES}

1. La vejiga neurogénica representa una alteración común es pacientes con lesión medular, que de no tratarse adecuadamente puede originar complicaciones urológicas importantes.

2. Los pacientes con lesión medular y vejiga neurogénica de alta presión-disinergia vésicoesfinteriana cursan con periodos de incontinencia vesical, que repercuten de manera importante en el enfoque social y psicológico, además de disminuir significativamente la calidad de vida.

3. La aplicación intravesical de toxina botulínica es un procedimiento con mínimas complicaciones y efectos adversos, lo que resulta en incremento significativo de la capacidad vesical y disminución del grado de incontinencia urinaria, con efectos benéficos a mediano y largo plazo.

4. Los pacientes con vejiga de alta presióndisinergia vesicoesfinteriana pueden beneficiarse con la aplicación intravesical de la toxina botulínica, pues permite facilitar el cateterismo limpio intermitente y realizar sus actividades cotidianas, reflejadas en mejor calidad de vida.

5. No se encontró diferencia significativa para estandarizar la aplicación intravesical de la toxina botulínica; por tanto, se requieren estudios adicionales, con mayor número de pacientes y evaluación de otro tipo de variables.

\section{Financiamiento}

Los autores no recibieron ningún patrocinio para llevar a cabo este estudio.

\section{Conflicto de interés}

Los autores declaran no tener conflicto de interés.

\section{REFERENCIAS}

1. Danforth TL, Ginsberg DA. Neurogenic lower urinary tract dysfunction. Urol Clin N Am 2014;41:445-452.

2. Jeong SJ, Cho SY, Oh SJ. Spinal cord/brain injury and the neurogenic bladder. Urol Clin N Am. 2010;37:537-546.

3. Middleton J, Ramakrishnan K, Cameron I. Management of the neurogenic bladder for adults with spinal cord injuries. $3^{\text {rd }}$ Ed. Sydney: NSW Agency for Clinical Innovation, 2014.

4. Jonas U, Castro-Díaz D, Bemelmans BLH, et al. Neurogenic voiding dysfunctions. Eur Urol 2003;44:I-XV.

5. Gormley EA. Urologic Complications of the neurogenic bladder. Urol Clin N Am 2010;37:601-607.

6. McGuire EJ. Urodynamics of the neurogenic bladder. Urol Clin N Am 2010; 37:507-516.

7. Patel AK, Patterson JM, Chapple CR. Botulinum toxin injections for neurogenic and idiopathic detrusor overactivity: A critical analysis of results. Eur Urol 2006;50:684-710.

8. Cameron AP. Pharmacologic therapy for the neurogenic bladder. Urol Clin N Am 2010;37:495-506.

9. Apostolidis A, Dasgupta P, Denys P, et al. Recommendations on the use of botulinum toxin in the treatment of lower urinary tract disorders and pelvic floor dysfunctions: A European Consensus Report. Eur Urol 2009;55:100-119.

10. Schurh B, Schulte-Baukloh H. Botulinum toxin in the treatment of neurogenic bladder in adults and children. Eur Urol Suppl 2006;5(11):679-684.

11. Neel KF, Soliman S, Salem M, et al. Botulinum a toxin: treatment for neuropathic noncompliant bladder. J Urol 2007;178(6):2593-2598.

12. Guzmán $\mathrm{S}$, Honeck $\mathrm{P}$, Weiss J, et al. Inyección de toxina botulínica en disfunción vesical neurogénica y no neurogénica. Arch Esp Urol 2005;58:651-655.

13. Kuo HC. Recovery of detrusor function after urethral botulinum a toxin injection in patients with idiopathic low detrusor contractility and voiding dysfunction. Urology 2007;69:57-62.

14. Duthie JB, Vincent $M$, Herbinson GP, et al. Botulinum toxin injections for adults with overactive bladder syndrome. The Cochrane Database Syst Rev 2011; 12: CD005493. doi: 10.1002/14651858.CD005493.pub3.

15. Karsenty $G$, Denys $P$, Amarenco $G$, et al. Botulinum toxin a (botox) intradetrusor injections in adults with neurogenic detrusor overactivity/neurogenic overactive bladder: a systematic literature review. Eur Urol 2008;53:275-287.

16. Liao YM, Kuo HC. Causes of failed urethral botulinum toxin a treatment for emptying failure. Urology 2007; 70(4): 763-766. 